\title{
Decision-Making Analysis Based on Fermatean Fuzzy Yager Aggregation Operators with Application in COVID-19 Testing Facility
}

\author{
Harish Garg $\mathbb{D}^{1},{ }^{1}$ Gulfam Shahzadi, ${ }^{2}$ and Muhammad Akram $\mathbb{i D}^{2}$ \\ ${ }^{1}$ School of Mathematics, Thapar Institute of Engineering and Technology, Deemed University, Patiala 147004, Punjab, India \\ ${ }^{2}$ Department of Mathematics, University of the Punjab, New Campus, Lahore 54590, Pakistan \\ Correspondence should be addressed to Harish Garg; harishg58iitr@gmail.com
}

Received 7 June 2020; Accepted 10 July 2020; Published 18 August 2020

Guest Editor: S. A. Edalatpanah

Copyright (c) 2020 Harish Garg et al. This is an open access article distributed under the Creative Commons Attribution License, which permits unrestricted use, distribution, and reproduction in any medium, provided the original work is properly cited.

\begin{abstract}
This research article is devoted to establish some general aggregation operators, based on Yager's t-norm and t-conorm, to cumulate the Fermatean fuzzy data in decision-making environments. The Fermatean fuzzy sets (FFSs), an extension of the orthopair fuzzy sets, are characterized by both membership degree (MD) and nonmembership degree (NMD) that enable them to serve as an excellent tool to represent inexact human opinions in the decision-making process. In this article, the valuable properties of the FFS are merged with the Yager operator to propose six new operators, namely, Fermatean fuzzy Yager weighted average (FFYWA), Fermatean fuzzy Yager ordered weighted average (FFYOWA), Fermatean fuzzy Yager hybrid weighted average (FFYHWA), Fermatean fuzzy Yager weighted geometric (FFYWG), Fermatean fuzzy Yager ordered weighted geometric (FFYOWG), and Fermatean fuzzy Yager hybrid weighted geometric (FFYHWG) operators. A comprehensive discussion is made to elaborate the dominant properties of the proposed operators. To verify the importance of the proposed operators, an MADM strategy is presented along with an application for selecting an authentic lab for the COVID-19 test. The superiorities of the proposed operators and limitations of the existing operators are discussed with the help of a comparative study. Moreover, we have explained comparison between the proposed theory and the Fermatean fuzzy TOPSIS method to check the accuracy and validity of the proposed operators. The influence of various values of the parameter in the Yager operator on decision-making results is also examined.
\end{abstract}

\section{Introduction}

Decision making (DM) plays a vital role in the practical life activities of human beings as it refers to a process that lays out all the options according to the assessment data of the decision makers and then selects the excellent one, mostly happening in our everyday lives. In the early era of social development, decision makers utilized the real numbers as a rule to offer their assessment information. As the multiattribute decision-making (MADM) problems are becoming complex, the experts cannot give exact real numbers to assess the alternatives. The ambiguities and imprecision of human judgments highlighted the deficiency of the crisp set theory. Therefore, Zadeh [1] laid the foundations of the fuzzy set (FS) theory for uncertain knowledge that permits the experts to describe their satisfaction level (membership degree) regarding performance of a member within the unit interval. The FSs provide the grounds to the uncertain assessments, but they were not adequate enough to describe the NMD. To overcome this deficiency of FSs, Atanassov [2] developed intuitionistic fuzzy sets (IFSs) which have both degrees of satisfaction and dissatisfaction that make them more superior and efficient than the traditional FSs. The IFSs accommodate more amount of vagueness in DM as they provide information both in favor (membership grade) and against (nonmembership grade) of the available alternatives. Along with all these advantages, there were some limitations is Atanassov's model as the sum of MD and NMD is restricted to unit interval. For example, if MD of an element in a set is 0.7 and NMD is 0.4 , then sum of these values is 
greater than 1. To handle such situations, Yager [3] established Pythagorean fuzzy sets (PFSs) with relaxed conditions that enable them to handle imprecise decisions efficiently. However, the PFS also has some limitations; if MD of an element in a set is 0.9 and NMD is 0.6 , then sum of square of these values is greater than 1 . Then, Yager [4] developed the theory of the $q$-rung orthopair fuzzy set ( $q$-ROFS) with condition that the sum of $q^{\text {th }}$ power of MD and NMD is bounded by 1. Recently, Senapati and Yager [5] gave the concept of FFS as a generalization of IFS and PFS.

The concept of aggregation operators (AOs) was introduced to get a unique value by the list of values. The theory of averaging operators under intuitionistic fuzzy (IF) environment was studied by $\mathrm{Xu}$ [6]. Xu and Yager [7] discussed geometric operators under IF environment. Li [8] studied the generalized ordered weighted averaging operators for IF data. The idea of induced geometric AOs under IF information was proposed by Wei [9]. The arithmetic and geometric operators were discussed in detail under Pythagorean fuzzy (PF) environment by Yager [10]. Peng and Yang [11] developed the fundamental properties of interval-valued PF aggregation operators. Zeng et al. [12] proposed a hybrid structure for PF MADM. Akram et al. [13] worked for the development of Pythagorean Dombi fuzzy AOs with applications. Shahzadi et al. [14] developed the theory of Yager AOs under PF data. Akram et al. $[15,16]$ proposed the group decision-making approaches with explanatory examples for the PF information. Many researchers gave more attention to the MADM based on the aggregation operators under different models of obscure knowledge, including IFS and PFS, [17-25]. Liu and Wang [26] discussed $q$-ROF weighted AOs. Dombi AOs under $q$-ROFS were defined by Jana et al. [27]. Liu et al. [28] studied $q$-ROF power Maclaurin symmetric mean operators. Liu and Liu [29] discussed $q$-ROF Bonferroni mean operators. Senapati and Yager [30] studied subtraction, division, and Fermatean arithmetic mean operations over FFS. The idea of Fermatean fuzzy (FF) weighted averaging/ geometric operators was also given by Senapati and Yager [31]. For other terminologies not discussed in the paper, the readers are referred to [32-39]. There are some incentives of this article:

(1) The judgment of perfect alternative is a difficult task under MADM environment when assessment data are simply illustrated by IF numbers and PF numbers which may prompt data mutilation. Therefore, we need a more general model to elaborate the potential of alternatives.

(2) Fermatean fuzzy sets, a remarkable extension of IFSs and PFSs, permit modeling of situations with more generality than IFSs and PFSs because these previous models fail to handle the situations where $\mu^{3}+\nu^{3} \leq 1$.

(3) As a prevalent set, Fermatean fuzzy numbers (FFNs) indicate extraordinary execution in providing vague, reliable, and inexact assessment information due to the modified and relaxed conditions. Therefore, FFNs might be the best approach for assessing the potential of alternatives.
(4) Yager AOs are straight forward, however, groundbreaking, approach for solving DM problems. Therefore, in this article, we aim to define Yager AOs in the FF context to deal with difficult MADM problems of choice.

(5) Yager AOs exhibit more precise results when applied to real-life MADM based on the FF data.

(6) The drawbacks and limitations of the existing operators are run over by the proposed operators as these operators are more general that work excellently not only for FF information but also for IF and PF data.

The major contributions of this research are as follows:

(1) The theory of Yager AOs is extended to FF numbers, and a thorough discussion is presented to analyze the important results and dominant properties of the proposed operators.

(2) An algorithm is proposed to deal complex practical MADM problems with FF data. The proposed algorithm is supported by an illustrative example for the selection of the most authentic lab for the COVID-19 test.

(3) The impact of the parameter's values on the proposed operators is explored to verify their authenticity.

(4) The consistency of the proposed approach is checked by conducting a comparative analysis with the existing operators and the FF TOPSIS method.

The remaining paper is composed as follows: Section 2 recalls the elementary notions of the FF and other existing models. In Section 3, we present Yager operations for Fermatean fuzzy numbers (FFNs) and establish some laws and aggregation operators. Section 4 gives an algorithm for MADM and discuss a numerical example in the field of medical based on FFNs. In Section 5, results are compared with existing operators to show the superiority and validity of the proposed theory. We have presented the comparison between the proposed theory and the FF TOPSIS method. Section 6 provides the conclusions about the proposed theory.

\section{Preliminaries}

In this section, we review some basic concepts on a fuzzy set.

Definition 1 (see [2]). An intuitionistic fuzzy set $I$ over the domain $\mathscr{J}$ is defined as

$$
I=\left\{\left\langle j, \mu_{I}(j), v_{I}(j)\right\rangle \mid j \in \mathscr{J}\right\},
$$

where $\mu_{I}: \mathscr{J} \longrightarrow[0,1]$ and $\nu_{I}: \mathscr{J} \longrightarrow[0,1]$ specify MD and NMD of an element $j \in \mathscr{F}$, respectively. For an element $j \in \mathcal{J}, \varpi_{I}(j)=1-\mu_{I}(j)-v_{I}(j)$ represents the indeterminacy degree $(\mathrm{InD})$.

Definition 2 (see [3]). A Pythagorean fuzzy set $P$ over the domain $\mathscr{J}$ is defined as 


$$
P=\left\{\left\langle j, \mu_{P}(j), v_{P}(j)\right\rangle\right\},
$$

where $\mu_{P}: \mathscr{J} \longrightarrow[0,1]$ and $\nu_{P}: \mathscr{J} \longrightarrow[0,1]$ specify $\mathrm{MD}$ and NMD of every element, respectively. $\varpi_{P}(j)=\sqrt{1-\left(\mu_{P}(j)\right)^{2}-\left(v_{P}(j)\right)^{2}}$ is InD.

Definition 3 (see [5]). A Fermatean fuzzy set $\mathscr{F}$ over the domain $\mathscr{J}$ is defined as

$$
\mathscr{F}=\left\{\left\langle j, \mu_{\mathscr{F}}(j), \nu_{\mathscr{F}}(j)\right\rangle\right\},
$$

where $\quad \mu_{\mathscr{F}}: \mathscr{J} \longrightarrow[0,1], \quad v_{\mathscr{F}}: \mathscr{J} \longrightarrow[0,1], \quad$ and $\varpi_{\mathscr{F}}(j)=\sqrt[3]{1-\left(\mu_{\mathscr{F}}(j)\right)^{3}-\left(\nu_{\mathscr{F}}(j)\right)^{3}}$ specify MD, NMD, and $\mathrm{InD}$, respectively. FFNs are components of the FFS.

Definition 4 (see [5]). The score function and accuracy function for FFN $\mathscr{F}=\left(\mu_{\mathscr{F}}, v_{\mathscr{F}}\right)$ are represented by

$$
\begin{array}{clll}
S(\mathscr{F})=\mu_{\mathscr{F}}^{3}-\nu_{\mathscr{F}}^{3}, & \text { where } & & S(\mathscr{F}) \in[-1,1], \\
H(\mathscr{F}) & =\mu_{\mathscr{F}}^{3}+\nu_{\mathscr{F}}^{3}, & \text { where } & H(\mathscr{F}) \in[0,1] .
\end{array}
$$

Definition 5 (see [5]). Consider two FFNs $\mathscr{F}_{1}=\left\langle\mu_{\mathscr{F}_{1}}, v_{\mathscr{F}_{1}}\right\rangle$ and $\mathscr{F}_{2}=\left\langle\mu_{\mathscr{F}_{2}}, v_{\mathscr{F}_{2}}\right\rangle$. Then,

(1) If $S\left(\mathscr{F}_{1}\right)<S\left(\mathscr{F}_{2}\right)$, then $\mathscr{F}_{1}<\mathscr{F}_{2}$

(2) If $S\left(\mathscr{F}_{1}\right)>S\left(\mathscr{F}_{2}\right)$, then $\mathscr{F}_{1}>\mathscr{F}_{2}$

(3) If $S\left(\mathscr{F}_{1}\right)=S\left(\mathscr{F}_{2}\right)$, then

(a) If $H\left(\mathscr{F}_{1}\right)<H\left(\mathscr{F}_{2}\right)$, then $\mathscr{F}_{1}<\mathscr{F}_{2}$

(b) If $H\left(\mathscr{F}_{1}\right)>H\left(\mathscr{F}_{2}\right)$, then $\mathscr{F}_{1}>\mathscr{F}_{2}$ (c) If $H\left(\mathscr{F}_{1}\right)=H\left(\mathscr{F}_{2}\right)$, then $\mathscr{F}_{1} \sim \mathscr{F}_{2}$

\section{Fermatean Fuzzy Numbers under Yager Operations}

This section addresses some operational laws and their operators for FFNs.

\subsection{Operational Laws for FFNs}

Definition 6. Let $\mathscr{F}_{1}=\left\langle\mu_{1}, v_{1}\right\rangle$ and $\mathscr{F}_{2}=\left\langle\mu_{2}, v_{2}\right\rangle$ be two FFNs, $\eta>0$ and $\xi>0$. Then, Yager $t$-norm and $t$-conorm operations of FFNs are

(1) $\mathscr{F}_{1} \oplus \mathscr{F}_{2}=\left\langle\sqrt[3]{\min \left(1,\left(\mu_{1}^{3 \eta}+\mu_{2}^{3 \eta}\right)^{1 / \eta}\right)}, \sqrt[3]{1-}\right.$ $\left.\min \left(1,\left(\left(1-v_{1}^{3}\right)^{\eta}+\left(1-v_{2}^{3}\right)^{\eta}\right)^{1 / \eta}\right)\right\rangle$

(2) $\mathscr{F}_{1} \otimes \mathscr{F}_{2}=\left\langle\sqrt[3]{1-\min \left(1,\left(\left(1-\mu_{1}^{3}\right)^{\eta}+\right.\right.}\right.$ $\left.\left.\left(1-\mu_{2}^{3}\right)^{\eta}\right)^{1 / \eta}\right), \sqrt[3]{\left.\min \left(1,\left(\nu_{1}^{3 \eta}+\nu_{2}^{3 \eta}\right)^{1 / \eta}\right)\right\rangle}$

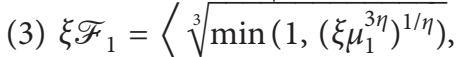
$\sqrt[3]{\left.1-\min \left(1,\left(\xi\left(1-\nu_{1}^{3}\right)^{\eta}\right)^{1 / \eta}\right)\right\rangle}$

(4) $\begin{aligned} & \mathscr{F}_{1}^{\xi}=\left\langle\sqrt[3]{1-\min \left(1,\left(\xi\left(1-\mu_{1}^{3}\right)^{\eta}\right)^{1 / \eta}\right)},\right. \\ &\left.\sqrt[3]{\min \left(1,\left(\xi \nu_{1}^{3 \eta}\right)^{1 / \eta}\right)}\right\rangle\end{aligned}$

Example 1. Let $\mathscr{F}_{1}=\langle 0.9,0.4\rangle$ and $\mathscr{F}_{2}=\langle 0.7,0.8\rangle$ be two FFNs, and then by using Definition 6 for $\eta=3, \xi=5$ they are:

$$
\begin{aligned}
\mathscr{F}_{1} \oplus \mathscr{F}_{2} & =\left\langle\sqrt[3]{\min \left(1,\left(0.9^{9}+0.7^{9}\right)^{1 / 3}\right)}, \sqrt[3]{1-\min \left(1,\left(\left(1-0.4^{3}\right)^{3}+\left(1-0.8^{3}\right)^{3}\right)^{1 / 3}\right)}\right\rangle=\langle 0.90,0.19\rangle, \\
\mathscr{F}_{1} \otimes \mathscr{F}_{2} & =\left\langle\sqrt[3]{1-\min \left(1,\left(\left(1-0.9^{3}\right)^{3}+\left(1-0.7^{3}\right)^{3}\right)^{1 / 3}\right)}, \sqrt[3]{\min \left(1,\left(0.4^{9}+0.8^{9}\right)^{1 / 3}\right)}\right\rangle=\langle 0.69,0.80\rangle, \\
5 \mathscr{F}_{1} & =\left\langle\sqrt[3]{\min \left(1,\left(5(0.9)^{9}\right)^{1 / 3}\right)}, \sqrt[3]{1-\min \left(1,\left(5\left(1-0.4^{3}\right)^{3}\right)^{1 / 3}\right)}\right\rangle=\langle 1,0\rangle, \\
\mathscr{F}_{1}^{5} & =\left\langle\sqrt[3]{1-\min \left(1,\left(5\left(1-0.9^{3}\right)^{3}\right)^{1 / 3}\right)}, \sqrt[3]{\min \left(1,\left(5(0.4)^{9}\right)^{1 / 3}\right)}\right\rangle=\langle 0.81,0.48\rangle .
\end{aligned}
$$

Theorem 1. Let $\mathscr{F}=\langle\mu, v\rangle, \mathscr{F}_{1}=\left\langle\mu_{1}, v_{1}\right\rangle, \mathscr{F}_{2}=\left\langle\mu_{2}, v_{2}\right\rangle$ be three FFNs, and then

(1) $\mathscr{F}_{1} \oplus \mathscr{F}_{2}=\mathscr{F}_{2} \oplus \mathscr{F}_{1}$

(2) $\mathscr{F}_{1} \otimes \mathscr{F}_{2}=\mathscr{F}_{1} \otimes \mathscr{F}_{2}$

(3) $\xi\left(\mathscr{F}_{1} \oplus \mathscr{F}_{2}\right)=\xi \mathscr{F}_{1} \oplus \xi \mathscr{F}_{2}$
(4) $\left(\xi_{1}+\xi_{2}\right) \mathscr{F}=\xi_{1} \mathscr{F} \oplus \xi_{2} \mathscr{F}$

(5) $\left(\mathscr{F}_{1} \otimes \mathscr{F}_{2}\right)^{\xi}=\mathscr{F}_{1}^{\xi} \otimes \mathscr{F}_{2}^{\xi}, \xi>0$

(6) $\mathscr{F}^{\xi_{1}} \otimes \mathscr{F}^{\xi_{2}}=\mathscr{F}^{\left(\xi_{1}+\xi_{2}\right)}, \xi_{1}, \xi_{2}>0$

Proof. For three FFNs $\mathscr{F}, \mathscr{F}_{1}, \mathscr{F}_{2}$ and $\xi, \xi_{1}, \xi_{2}>0$, by Definition 6, 


$$
\begin{aligned}
& \mathscr{F}_{1} \oplus \mathscr{F}_{2}=\left\langle\sqrt[3]{\min \left(1,\left(\mu_{1}^{3 \eta}+\mu_{2}^{3 \eta}\right)^{1 / \eta}\right)}, \sqrt[3]{1-\min \left(1,\left(\left(1-v_{1}^{3}\right)^{\eta}+\left(1-v_{2}^{3}\right)^{\eta}\right)^{1 / \eta}\right)}\right\rangle \\
& =\left\langle\sqrt[3]{\min \left(1,\left(\mu_{1}^{3 \eta}+\mu_{2}^{3 \eta}\right)^{1 / \eta}\right)}, \sqrt[3]{1-\min \left(1,\left(\left(1-v_{1}^{3}\right)^{\eta}+\left(1-v_{2}^{3}\right)^{\eta}\right)^{1 / \eta}\right)}\right\rangle \\
& =\mathscr{F}_{2} \oplus \mathscr{F}_{1} \text {, } \\
& \mathscr{F}_{1} \otimes \mathscr{F}_{2}=\left\langle\sqrt[3]{1-\min \left(1,\left(\left(1-\mu_{1}^{3}\right)^{\eta}+\left(1-\mu_{2}^{3}\right)^{\eta}\right)^{1 / \eta}\right)}, \sqrt[3]{\min \left(1,\left(v_{1}^{3 \eta}+v_{2}^{3 \eta}\right)^{1 / \eta}\right)}\right\rangle \\
& =\left\langle\sqrt[3]{1-\min \left(1,\left(\left(1-\mu_{2}^{3}\right)^{\eta}+\left(1-\mu_{1}^{3}\right)^{\eta}\right)^{1 / \eta}\right)}, \sqrt[3]{\min \left(1,\left(v_{2}^{3 \eta}+v_{1}^{3 \eta}\right)^{1 / \eta}\right)}\right\rangle \\
& =\mathscr{F}_{2} \otimes \mathscr{F}_{1} \text {, } \\
& \xi\left(\mathscr{F}_{1} \oplus \mathscr{F}_{2}\right)=\xi\left\langle\sqrt[3]{\min \left(1,\left(\mu_{1}^{3 \eta}+\mu_{2}^{3 \eta}\right)^{1 / \eta}\right)}, \sqrt[3]{1-\min \left(1,\left(\left(1-v_{1}^{3}\right)^{\eta}+\left(1-v_{2}^{3}\right)^{\eta}\right)^{1 / \eta}\right)}\right\rangle \\
& =\left\langle\sqrt[3]{\min \left(1,\left(\xi \mu_{1}^{3 \eta}+\xi \mu_{2}^{3 \eta}\right)^{1 / \eta}\right)}, \sqrt[3]{1-\min \left(1,\left(\xi\left(1-\nu_{1}^{3}\right)^{\eta}+\xi\left(1-v_{2}^{3}\right)^{\eta}\right)^{1 / \eta}\right)}\right\rangle, \\
& \xi \mathscr{F}_{1} \oplus \xi \mathscr{F}_{2}=\left\langle\sqrt[3]{\min \left(1,\left(\xi \mu_{1}^{3 \eta}\right)^{1 / \eta}\right)}, \sqrt[3]{1-\min \left(1,\left(\xi\left(1-v_{1}^{3}\right)^{\eta}\right)^{1 / \eta}\right)}\right\rangle \oplus\left\langle\sqrt[3]{\min \left(1,\left(\xi \mu_{2}^{3 \eta}\right)^{1 / \eta}\right)}, \sqrt[3]{1-\min \left(1,\left(\xi\left(1-\nu_{2}^{3}\right)^{\eta}\right)^{1 / \eta}\right)}\right\rangle \\
& =\left\langle\sqrt[3]{\min \left(1,\left(\xi \mu_{1}^{3 \eta}+\xi \mu_{2}^{3 \eta}\right)^{1 / \eta}\right)}, \sqrt[3]{1-\min \left(1,\left(\xi\left(1-v_{1}^{3}\right)^{\eta}+\xi\left(1-v_{2}^{3}\right)^{\eta}\right)^{1 / \eta}\right)}\right\rangle \\
& =\xi\left(\mathscr{F}_{1} \oplus \mathscr{F}_{2}\right) \text {, } \\
& \xi_{1} \mathscr{F} \oplus \xi_{2} \mathscr{F}=\left\langle\sqrt[3]{\min \left(1,\left(\xi_{1} \mu^{3 \eta}\right)^{1 / \eta}\right)}, \sqrt[3]{1-\min \left(1,\left(\xi_{1}\left(1-v^{3}\right)^{\eta}\right)^{1 / \eta}\right)}\right\rangle \oplus\left\langle\sqrt[3]{\min \left(1,\left(\xi_{2} \mu^{3 \eta}\right)^{1 / \eta}\right)}, \sqrt[3]{1-\min \left(1,\left(\xi_{2}\left(1-v^{3}\right)^{\eta}\right)^{1 / \eta}\right)}\right\rangle \\
& =\left\langle\sqrt[3]{\min \left(1,\left(\left(\xi_{1}+\xi_{2}\right) \mu^{3 \eta}\right)^{1 / \eta}\right)}, \sqrt[3]{1-\min \left(1,\left(\left(\xi_{1}+\xi_{2}\right)\left(1-v^{3}\right)^{\eta}\right)^{1 / \eta}\right)}\right\rangle \\
& =\left(\xi_{1}+\xi_{2}\right) \mathscr{F} \text {. }
\end{aligned}
$$

In this same way, other properties can be done.

\subsection{Fermatean Fuzzy Yager Hybrid Weighted Arithmetic} Operators. Yager weighted arithmetic operators under FF environment are discussed here.

Definition 7. Let $\mathscr{F}_{b}=\left\langle\mu_{b}, v_{b}\right\rangle(b=1,2, \ldots, t)$ be a collection of FFNs. The FFYWA operator is a function $\mathbb{Q}^{t} \longrightarrow \mathbb{Q}$ s.t.

$$
\operatorname{FFYWA}_{\alpha}\left(\mathscr{F}_{1}, \mathscr{F}_{2}, \ldots, \mathscr{F}_{t}\right)=\bigoplus_{b=1}^{t}\left(\alpha_{b} \mathscr{F}_{b}\right)
$$

where $\alpha=\left(\alpha_{1}, \alpha_{2}, \ldots, \alpha_{t}\right)^{T}$ is the weight vector (WV) of $\mathscr{F}_{b}$ with $\alpha_{b}>0$ and $\sum_{b=1}^{t} \alpha_{b}=1$.

Theorem 2. Let $\mathscr{F}_{b}=\left\langle\mu_{b}, v_{b}\right\rangle(b=1,2, \ldots, t)$ be a collection of FFNs, and then the aggregated value of them by the FFYWA operation is an FFN and

$$
\left.\operatorname{FFYWA}_{\alpha}\left(\mathscr{F}_{1}, \mathscr{F}_{2}, \ldots, \mathscr{F}_{t}\right)=\left\langle\sqrt[3]{\min \left(1,\left(\sum_{b=1}^{t}\left(\alpha_{b} \mu_{b}^{3 \eta}\right)\right)^{1 / \eta}\right.}\right) \sqrt[3]{1-\min \left(1,\left(\sum_{b=1}^{t}\left(\alpha_{b}\left(1-v_{b}^{3}\right)^{\eta}\right)\right)^{1 / \eta}\right)}\right\rangle
$$


Proof. To prove this result, use mathematical induction.

(i) When $t=2$,

$$
\begin{aligned}
& \alpha_{1} \mathscr{F}_{1}=\left\langle\sqrt[3]{\min \left(1,\left(\alpha_{1} \mu_{1}^{3 \eta}\right)^{1 / \eta}\right)}, \sqrt[3]{1-\min \left(1,\left(\alpha_{1}\left(1-v_{1}^{3}\right)^{\eta}\right)^{1 / \eta}\right)}\right\rangle, \\
& \alpha_{2} \mathscr{F}_{2}=\left\langle\sqrt[3]{\min \left(1,\left(\alpha_{2} \mu_{2}^{3 \eta}\right)^{1 / \eta}\right)}, \sqrt[3]{1-\min \left(1,\left(\alpha_{2}\left(1-v_{2}^{3}\right)^{\eta}\right)^{1 / \eta}\right)}\right\rangle .
\end{aligned}
$$

Therefore,

$$
\begin{aligned}
\alpha_{1} \mathscr{F}_{1} \oplus \alpha_{2} \mathscr{F}_{2} & =\left\langle\sqrt[3]{\min \left(1,\left(\alpha_{1} \mu_{1}^{3 \eta}\right)^{1 / \eta}\right)}, \sqrt[3]{1-\min \left(1,\left(\alpha_{1}\left(1-v_{1}^{3}\right)^{\eta}\right)^{1 / \eta}\right)}\right\rangle \\
& \oplus\left\langle\sqrt[q]{\min \left(1,\left(\alpha_{2} \mu_{2}^{3 \eta}\right)^{1 / \eta}\right)}, \sqrt[3]{1-\min \left(1,\left(\alpha_{2}\left(1-v_{2}^{3}\right)^{\eta}\right)^{1 / \eta}\right)}\right\rangle \\
& =\left\langle\sqrt[3]{\min \left(1,\left(\alpha_{1} \mu_{1}^{3 \eta}+\alpha_{2} \mu_{2}^{3 \eta}\right)^{1 / \eta}\right)}, \sqrt[3]{1-\min \left(1,\left(\alpha_{1}\left(1-\nu_{1}^{3}\right)^{\eta}+\alpha_{2}\left(1-v_{2}^{3}\right)\right)^{1 / \eta}\right)}\right\rangle \\
& \left.\left.=\left\langle\sqrt[3]{\min \left(1,\left(\sum_{b=1}^{2}\left(\alpha_{b} \mu_{b}^{3 \eta}\right)\right)^{1 / \eta}\right.}\right), \sqrt[3]{1-\min \left(1,\left(\sum_{b=1}^{2}\left(\alpha_{b}\left(1-v_{b}^{3}\right)^{\eta}\right)\right)^{1 / \eta}\right)}\right)\right\rangle
\end{aligned}
$$

Hence, equation (8) is true for $t=2$.

(ii) Let equation (8) holds for $t=k$,

$$
\begin{aligned}
\operatorname{FFYWA}_{\alpha}\left(\mathscr{F}_{1}, \mathscr{F}_{2}, \ldots, \mathscr{F}_{k}\right) & =\bigoplus_{b=1}^{k}\left(\alpha_{b} \mathscr{F}_{b}\right) \\
& \left.=\left\langle\sqrt[3]{\min \left(1,\left(\sum_{b=1}^{k}\left(\alpha_{b} \mu_{b}^{3 \eta}\right)\right)^{1 / \eta}\right.}\right), \sqrt[3]{1-\min \left(1,\left(\sum_{b=1}^{k}\left(\alpha_{b}\left(1-v_{b}^{3}\right)^{\eta}\right)\right)^{1 / \eta}\right)}\right\rangle
\end{aligned}
$$

Now, for $t=k+1$,

$$
\begin{aligned}
\operatorname{FFYWA}_{\alpha}\left(\mathscr{F}_{1}, \mathscr{F}_{2}, \ldots, \mathscr{F}_{k+1}\right)= & \left\langle\sqrt[3]{\min \left(1,\left(\sum_{b=1}^{k}\left(\alpha_{b} \mu_{b}^{3 \eta}\right)\right)^{1 / \eta}\right.}, \sqrt[3]{\left.1-\min \left(1,\left(\sum_{b=1}^{k}\left(\alpha_{b}\left(1-v_{b}^{3}\right)^{\eta}\right)\right)^{1 / \eta}\right)\right\rangle}\right. \\
& \left\langle\sqrt[3]{\min \left(1,\left(\alpha_{k+1} \mu_{k+1}^{3 \eta}\right)^{1 / \eta}\right)}, \sqrt[3]{\left.1-\min \left(1,\left(\alpha_{k+1}\left(1-v_{k+1}^{3}\right)^{\eta}\right)^{1 / \eta}\right)\right\rangle}\right\rangle \\
= & \left.\left\langle\sqrt[3]{\min \left(1,\left(\sum_{b=1}^{k+1}\left(\alpha_{b} \mu_{b}^{3 \eta}\right)\right)^{1 / \eta}\right.}\right), \sqrt[3]{1-\min \left(1,\left(\sum_{b=1}^{k+1} \alpha_{b}\left(1-v_{b}^{3}\right)^{\eta}\right)^{1 / \eta}\right)}\right\rangle
\end{aligned}
$$

Hence, equation (8) is true for $t=k+1$. Thus, equation (8) is true, $\forall t$.
Example 2. Let $\mathscr{F}_{1}=\langle 0.6,0.9\rangle, \mathscr{F}_{2}=\langle 0.8,0.7\rangle, \mathscr{F}_{3}=$ $\langle 0.7,0.6\rangle$, and $\mathscr{F}_{4}=\langle 0.9,0.7\rangle$ be FFNs with a WV 
$\alpha=(0.2,0.3,0.2,0.3)^{T}$ and $\eta=2$. By Theorem 2, the aggregated value of FFNs is

$$
\begin{aligned}
& \operatorname{FFYWA}_{\alpha}\left(\mathscr{F}_{1}, \mathscr{F}_{2}, \mathscr{F}_{3}, \mathscr{F}_{4}\right)=\bigoplus_{b=1}^{4}\left(\alpha_{b} \mathscr{F}_{b}\right) \\
& =\left\langle\sqrt[3]{\min \left(1,\left(\sum_{b=1}^{4}\left(\alpha_{b} \mu_{b}^{3 \eta}\right)\right)^{1 / \eta}\right)}, \sqrt[3]{1-\min \left(1,\left(\sum_{b=1}^{4}\left(\alpha_{b}\left(1-v_{b}^{3}\right)^{\eta}\right)\right)^{1 / \eta}\right)}\right\rangle \\
& =\left\langle\sqrt[3]{\min \left(1,\left(0.2(0.6)^{6}+0.3(0.8)^{6}+0.2(0.7)^{6}+0.3(0.9)^{6}\right)^{1 / 2}\right)},\right. \\
& \left.\sqrt[3]{1-\min \left(1,\left(0.2\left(1-0.9^{3}\right)^{2}+0.3\left(1-0.7^{3}\right)^{2}+0.2\left(1-0.6^{3}\right)^{2}+0.2\left(1-0.7^{3}\right)^{2}\right)^{1 / 2}\right)}\right\rangle \\
& =\langle 0.80,0.74\rangle \text {. }
\end{aligned}
$$

Theorem 3 (idempotency). If all FFNs are identical, i.e., Proof $\mathscr{F}_{b}=\mathscr{F}$, then

$$
\operatorname{FFYWA}\left(\mathscr{F}_{1}, \mathscr{F}_{2}, \ldots, \mathscr{F}_{t}\right)=\mathscr{F} \text {. }
$$

$$
\begin{aligned}
\operatorname{FFYWA}_{\alpha}\left(\mathscr{F}_{1}, \mathscr{F}_{2}, \ldots, \mathscr{F}_{t}\right) & =\bigoplus_{b=1}^{t}\left(\alpha_{b} \mathscr{F}_{b}\right) \\
& \left.=\left\langle\sqrt[3]{\min \left(1,\left(\sum_{b=1}^{t}\left(\alpha_{b} \mu_{b}^{3 \eta}\right)\right)^{1 / \eta}\right.}\right), \sqrt[3]{1-\min \left(1,\left(\sum_{b=1}^{t}\left(\alpha_{b}\right)\left(1-v_{b}^{3}\right)^{\eta}\right)^{1 / \eta}\right)}\right\rangle \\
& =\left\langle\sqrt[3]{\min \left(1,\left(\mu^{3 \eta}\right)^{1 / \eta}\right)}, \sqrt[3]{1-\min \left(1,\left(\left(1-\nu^{3}\right)^{\eta}\right)^{1 / \eta}\right)}\right\rangle \\
& =\left\langle\sqrt[3]{\min \left(1, \mu^{3}\right)}, \sqrt[3]{1-\min \left(1, t\left(1-\nu^{3}\right)\right)},\right\rangle \\
& =\langle\mu, \nu\rangle \\
& =\mathscr{F}
\end{aligned}
$$

Theorem 4 (boundedness). Let $\mathscr{F}_{b}=\left(\mu_{b}, v_{b}\right)$ be a collection of FFNs. Let $\mathscr{F}^{-}=\min \left(\mathscr{F}_{1}, \mathscr{F}_{2}, \ldots, \mathscr{F}_{t}\right)$ and $\mathscr{F}^{+}=\max \left(\mathscr{F}_{1}, \mathscr{F}_{2}, \ldots, \mathscr{F}_{t}\right)$. Then,

$$
\mathscr{F}^{-} \leq \operatorname{FFYWA}\left(\mathscr{F}_{1}, \mathscr{F}_{2}, \ldots, \mathscr{F}_{t}\right) \leq \mathscr{F}^{+} .
$$

Proof. Suppose that $\mathscr{F}^{-}=\min \left(\mathscr{F}_{1}, \mathscr{F}_{2}, \ldots, \mathscr{F}_{t}\right)=\left(\mu^{-}, v^{-}\right)$ and $\quad \mathscr{F}^{+}=\max \left(\mathscr{F}_{1}, \mathscr{F}_{2}, \ldots, \mathscr{F}_{t}\right)=\left(\mu^{+}, \nu^{+}\right)$, where $\mu^{-}=\min \left(\mu_{b}\right), \quad \nu^{-}=\max \left(\nu_{b}\right), \quad \mu^{+}=\max \left(\mu_{b}\right), \quad$ and $\nu^{+}=\min \left(\nu_{b}\right)$. The inequalities for membership value are

$$
\sqrt[3]{\min \left(1,\left(\sum_{b=1}^{t}\left(\alpha_{b} \mu^{-3 \eta}\right)\right)^{1 / \eta}\right)} \leq \sqrt[3]{\min \left(1,\left(\sum_{b=1}^{t}\left(\alpha_{b} \mu_{b}^{3 \eta}\right)\right)^{1 / \eta}\right.} \leq \sqrt[3]{\min \left(1,\left(\sum_{b=1}^{t}\left(\alpha_{b} \mu^{+3^{3 \eta}}\right)\right)^{1 / \eta}\right)} .
$$

Similarly, for nonmembership value,

$$
\sqrt[3]{1-\min \left(1,\left(\sum_{b=1}^{t}\left(\alpha_{b}\left(1-v^{+^{3}}\right)^{\eta}\right)\right)^{1 / \eta}\right)} \leq \sqrt[3]{1-\min \left(1,\left(\sum_{b=1}^{t}\left(\alpha_{b}\left(1-v_{b}^{3}\right)^{\eta}\right)\right)^{1 / \eta}\right)} \leq \sqrt[3]{1-\min \left(1,\left(\sum_{b=1}^{t}\left(\alpha_{b}\left(1-v^{-3}\right)^{\eta}\right)\right)^{1 / \eta}\right)}
$$


Therefore, $\mathscr{F}^{-} \leq \operatorname{FFYWA}\left(\mathscr{F}_{1}, \mathscr{F}_{2}, \ldots, \mathscr{F}_{t}\right) \leq \mathscr{F}^{+}$

Theorem 5 (monotonicity). Let $\mathscr{F}_{b}^{\prime}=\left\{\mathscr{F}_{1}^{\prime}, \mathscr{F}_{2}^{\prime}, \ldots, \mathscr{F}_{t}^{\prime}\right\}$ and $\mathscr{F}_{b}=\left\{\mathscr{F}_{1}, \mathscr{F}_{2}, \ldots, \mathscr{F}_{t}\right\}$ be two collections of FFNs. If $\mu_{b}^{\prime} \leq \mu_{b}$ and $\nu_{b}^{\prime} \geq v_{b}, \forall b$. Then,

$$
\operatorname{FFYWA}\left(\mathscr{F}_{1}^{\prime}, \mathscr{F}_{2}^{\prime}, \ldots, \mathscr{F}_{t}^{\prime}\right) \leq \operatorname{FFYWA}\left(\mathscr{F}_{1}, \mathscr{F}_{2}, \ldots, \mathscr{F}_{t}\right) \text {. }
$$

Proof. Let $\operatorname{FFYWA}\left(\mathscr{F}_{1}^{\prime}, \mathscr{F}_{2}^{\prime}, \ldots, \mathscr{F}_{t}^{\prime}\right)=\left(\mathscr{G}^{\prime}, \mathscr{K}^{\prime}\right)$ and $\operatorname{FFYWA}\left(\mathscr{F}_{1}, \mathscr{F}_{2}, \ldots, \mathscr{F}_{t}\right)=(\mathscr{G}, \mathscr{K})$. First, we will show that $\mathscr{G}^{\prime} \leq \mathscr{G}$. As $\mu_{b}^{\prime} \leq \mu_{b}, \mu_{b}^{\prime 3} \leq \mu_{b}^{3}$. Moreover,

$$
\begin{gathered}
\left(\sum_{b=1}^{t}\left(\alpha_{b} \mu_{b}^{\prime 3 \eta}\right)\right)^{1 / \eta} \leq\left(\sum_{b=1}^{t}\left(\alpha_{b} \mu_{b}^{3 \eta}\right)\right)^{1 / \eta} \\
\min \left(1,\left(\sum_{b=1}^{t}\left(\alpha_{b} \mu_{b}^{33 \eta}\right)\right)^{1 / \eta}\right) \leq \min \left(1,\left(\sum_{b=1}^{t}\left(\alpha_{b} \mu_{b}^{3 \eta}\right)\right)^{1 / \eta}\right) \\
\sqrt[3]{\min \left(1,\left(\sum_{b=1}^{t}\left(\alpha_{b} \mu_{b}^{3 \eta}\right)\right)^{1 / \eta}\right)} \leq \sqrt[3]{\min \left(1,\left(\sum_{b=1}^{t}\left(\alpha_{b} \mu_{b}^{3 \eta}\right)\right)^{1 / \eta}\right) .}
\end{gathered}
$$

Therefore, $\mathscr{G}^{\prime} \leq \mathscr{G}$. Similarly, we can show that $\mathscr{K}^{\prime} \geq \mathscr{K}$. Hence, $\quad \operatorname{FFYWA}\left(\mathscr{F}_{1}^{\prime}, \mathscr{F}_{2}^{\prime}, \ldots, \mathscr{F}_{t}^{\prime}\right) \leq \operatorname{FFYWA}\left(\mathscr{F}_{1}, \mathscr{F}_{2}, \ldots\right.$, $\left.\mathscr{F}_{t}\right)$.

Theorem 6 (reducibility). Let $\mathscr{F}_{b}=\left(\mu_{b}, v_{b}\right)(b=1,2, \ldots, t)$ be a collection of FFNs with corresponding $W V$ $\alpha=\left(\alpha_{1}, \alpha_{2}, \ldots, \alpha_{t}\right)^{T}=(1 / t, 1 / t, \ldots, 1 / t)^{T}$. Then, the FFYWA operator is

$$
\operatorname{FFYWA}_{\alpha}\left(\mathscr{F}_{1}, \mathscr{F}_{2}, \ldots, \mathscr{F}_{t}\right)=\left\langle\sqrt[3]{\min \left(1,\left(\frac{1}{t} \sum_{b=1}^{t}\left(\mu_{b}^{3 \eta}\right)\right)^{1 / \eta}\right)}, \sqrt[3]{1-\min \left(1,\left(\frac{1}{t} \sum_{b=1}^{t}\left(1-v_{b}^{3}\right)^{\eta}\right)^{1 / \eta}\right)}\right\rangle .
$$

We now define the FFYOWA operators.

Definition 8. Let $\mathscr{F}_{b}=\left\langle\mu_{b}, v_{b}\right\rangle(b=1,2, \ldots, t)$ be a collection of FFNs with WV $\alpha=\left(\alpha_{1}, \alpha_{2}, \ldots, \alpha_{t}\right)^{T}$ with $\alpha_{b}>0$ and $\sum_{b=1}^{t} \alpha_{b}=1$. The FFYOWA operator is a function $\mathbb{Q}^{t} \longrightarrow \mathbb{Q}$ s.t.

$$
\operatorname{FFYOWA}_{\alpha}\left(\mathscr{F}_{1}, \mathscr{F}_{2}, \ldots, \mathscr{F}_{t}\right)=\bigoplus_{b=1}^{t}\left(\alpha_{b} \mathscr{F}_{\varrho(b)}\right),
$$

where $(\varrho(1), \varrho(2), \ldots, \varrho(t)) \quad$ is permutation of $(b=1,2, \ldots, t)$ s.t. $\mathscr{F}_{\varrho(b-1)} \geq \mathscr{F}_{\varrho(b)}, \forall b=1,2, \ldots, t$.

Theorem 7. The aggregated value by applying the FFYOWA operator is also an FFN and given by

$$
\operatorname{FFYOWA}_{\alpha}\left(\mathscr{F}_{1}, \mathscr{F}_{2}, \ldots, \mathscr{F}_{t}\right)=\left\langle\sqrt[3]{\min \left(1,\left(\sum_{b=1}^{t}\left(\alpha_{b} \mu_{\varrho(b)}^{3 \eta}\right)\right)^{1 / \eta}\right)}, \sqrt[3]{1-\min \left(1,\left(\sum_{b=1}^{t}\left(\alpha_{b}\left(1-v_{\varrho(b)}^{3}\right)^{\eta}\right)\right)^{1 / \eta}\right)}\right\rangle .
$$

Example 3. Let $\mathscr{F}_{1}=\langle 0.8,0.7\rangle, \mathscr{F}_{2}=\langle 0.6,0.7\rangle, \mathscr{F}_{3}=$ $\langle 0.6,0.8\rangle$, and $\mathscr{F}_{4}=\langle 0.9,0.4\rangle$ be FFNs with a WV $\alpha=(0.1,0.3,0.3,0.3)^{T}$ and $\eta=2$. Then,

$$
\begin{aligned}
& \mathcal{S}\left(\mathscr{F}_{1}\right)=\frac{1}{2}\left(1+0.8^{3}+0.7^{3}\right)=0.93, \\
& \mathcal{S}\left(\mathscr{F}_{2}\right)=\frac{1}{2}\left(1+0.6^{3}+0.7^{3}\right)=0.78, \\
& \mathcal{S}\left(\mathscr{F}_{3}\right)=\frac{1}{2}\left(1+0.6^{3}+0.8^{3}\right)=0.86, \\
& \mathcal{S}\left(\mathscr{F}_{4}\right)=\frac{1}{2}\left(1+0.9^{3}+0.4^{3}\right)=0.90 .
\end{aligned}
$$

Since $\mathcal{S}\left(\mathscr{F}_{1}\right)>\mathcal{S}\left(\mathscr{F}_{4}\right)>\mathcal{S}\left(\mathscr{F}_{3}\right)>\mathcal{S}\left(\mathscr{F}_{2}\right)$,

$$
\begin{aligned}
& \mathscr{F}_{\varrho(1)}=\mathscr{F}_{1}=\langle 0.8,0.7\rangle, \\
& \mathscr{F}_{\varrho(2)}=\mathscr{F}_{4}=\langle 0.6,0.7\rangle, \\
& \mathscr{F}_{\varrho(3)}=\mathscr{F}_{3}=\langle 0.6,0.8\rangle, \\
& \mathscr{F}_{\varrho(4)}=\mathscr{F}_{2}=\langle 0.9,0.4\rangle .
\end{aligned}
$$

Thus, by applying the FFYOWA operator, 


$$
\begin{aligned}
\operatorname{FFYOWA}_{\alpha}\left(\mathscr{F}_{1}, \mathscr{F}_{2}, \mathscr{F}_{3}, \mathscr{F}_{4}\right) & =\bigoplus_{b=1}^{4}\left(\alpha_{b} \mathscr{F}_{\varrho(b)}\right) \\
& =\left\langle\sqrt[3]{\min \left(1,\left(\sum_{b=1}^{4}\left(\alpha_{b} \mu_{\varrho(b)}^{2 \eta}\right)\right)^{1 / \eta}\right)}, \sqrt[3]{1-\min \left(1,\left(\sum_{b=1}^{4}\left(\alpha_{b}\left(1-v_{\varrho(b)}^{2}\right)^{\eta}\right)\right)^{1 / \eta}\right)}\right\rangle \\
& =\left\langle\sqrt[3]{\min \left(1,\left(0.1(0.8)^{6}+0.3(0.6)^{6}+0.3(0.6)^{6}+0.3(0.9)^{6}\right)^{1 / 2}\right), \sqrt[3]{1-\min \left(1,\left(0.1\left(1-0.7^{3}\right)^{2}+0.3\left(1-0.7^{3}\right)^{2}+0.3\left(1-0.8^{3}\right)^{2}+0.3\left(1-0.4^{3}\right)^{2}\right)^{1 / 2}\right)}}\right\rangle \\
& =\langle 0.77,0.66\rangle .
\end{aligned}
$$

We give some statements without proofs.

Theorem 8 (idempotency). If all FFNs are identical, i.e., $\mathscr{F}_{b}=\mathscr{F}$, then

$$
\operatorname{FFYOWA}\left(\mathscr{F}_{1}, \mathscr{F}_{2}, \ldots, \mathscr{F}_{t}\right)=\mathscr{F} .
$$

Theorem 9 (boundedness). Let $\mathscr{F}_{b}=\left(\mu_{b}, \nu_{b}\right)$ be a collection of FFNs. Let $\mathscr{F}^{-}=\min \left(\mathscr{F}_{1}, \mathscr{F}_{2}, \ldots, \mathscr{F}_{t}\right) \quad$ and $\mathscr{F}^{+}=\max \left(\mathscr{F}_{1}, \mathscr{F}_{2}, \ldots, \mathscr{F}_{t}\right)$. Then,

$$
\mathscr{F}^{-} \leq \operatorname{FFYOWA}\left(\mathscr{F}_{1}, \mathscr{F}_{2}, \ldots, \mathscr{F}_{t}\right) \leq \mathscr{F}^{+} .
$$

Theorem 10 (monotonicity). Let $\mathscr{F}_{b}^{\prime}=\left\{\mathscr{F}_{1}^{\prime}, \mathscr{F}_{2}^{\prime}, \ldots, \mathscr{F}_{t}^{\prime}\right\}$ and $\mathscr{F}_{b}=\left\{\mathscr{F}_{1}, \mathscr{F}_{2}, \ldots, \mathscr{F}_{t}\right\}$ be two collections of FFNs. If $\mu_{b}^{\prime} \leq \mu_{b}$ and $\nu_{b}^{\prime} \geq v_{b}, \forall b$. Then,

$\operatorname{FFYOWA}\left(\mathscr{F}_{1}^{\prime}, \mathscr{F}_{2}^{\prime}, \ldots, \mathscr{F}_{t}^{\prime}\right) \leq \operatorname{FFYOWA}\left(\mathscr{F}_{1}, \mathscr{F}_{2}, \ldots, \mathscr{F}_{t}\right)$.

Theorem 11. Let $\mathscr{F}_{b}=\left(\mu_{b}, v_{b}\right)(b=1,2, \ldots, t)$ be a collection of FFNs with corresponding $W V \quad \alpha=\left(\alpha_{1}, \alpha_{2}, \ldots\right.$, $\left.\alpha_{t}\right)^{T}=(1 / t, 1 / t, \ldots, 1 / t)^{T}$. Then, the FFYOWA operator is

$$
\operatorname{FFYOWA}_{\alpha}\left(\mathscr{F}_{1}, \mathscr{F}_{2}, \ldots, \mathscr{F}_{t}\right)=\left\langle\sqrt[3]{\min \left(1, \frac{1}{t}\left(\sum_{b=1}^{t}\left(\mu_{b}^{3 \eta}\right)\right)^{1 / \eta}\right)}, \sqrt[3]{1-\min \left(1, \frac{1}{t}\left(\sum_{b=1}^{t}\left(1-v_{b}^{3}\right)^{\eta}\right)^{1 / \eta}\right)}\right\rangle .
$$

We now define the FFHWA operators.

$$
\begin{aligned}
& \operatorname{FFYHWA}_{\alpha}\left(\mathscr{F}_{1}, \mathscr{F}_{2}, \ldots, \mathscr{F}_{t}\right)=\bigoplus_{b=1}^{t}\left(\alpha_{b} \dot{\mathscr{F}}_{\varrho(b)}\right) \\
& =\left\langle\sqrt[3]{\min \left(1,\left(\sum_{b=1}^{t}\left(\alpha_{b} \dot{\mu}_{\varrho(b)}^{3 \eta}\right)\right)^{1 / \eta}\right)}, \sqrt[3]{1-\min \left(1,\left(\sum_{b=1}^{t}\left(\alpha_{b}\left(1-\dot{v}_{\varrho(b)}^{3}\right)^{\eta}\right)\right)^{1 / \eta}\right)}\right\rangle,
\end{aligned}
$$

where $\dot{\mathscr{F}}_{o(b)}$ is the $b$ th biggest weighted Fermatean fuzzy values $\dot{\mathscr{F}}_{b}\left(\dot{\mathscr{F}}_{b}=t \alpha_{b} \mathscr{F}_{b}, b=1,2, \ldots, t\right)$ and $t$ is the balancing coefficient.

Remark 1. For $\alpha=(1 / t, 1 / t, \ldots, 1 / t)^{T}$, FFYWA and FFYOWA operators are a particular example of the FFYHWA operator. Thus, the FFYHWA operator is a generalization of both operators.

3.3. Fermatean Fuzzy Yager Hybrid Weighted Geometric Operators. Yager weighted geometric operators are discussed here for FF data.
Definition 9. A FFYHWA is a function $\mathbb{Q}^{t} \longrightarrow \mathbb{Q}$, with correlated WV $\alpha=\left(\alpha_{1}, \alpha_{2}, \ldots, \alpha_{t}\right)^{T}$ with $\alpha_{b}>0$ and $\sum_{b=1}^{t} \alpha_{b}=1$ s.t.
Definition 10. Let $\mathscr{F}_{b}=\left\langle\mu_{b}, \nu_{b}\right\rangle(b=1,2, \ldots, t)$ be a number of FFNs. The FFYWG operator is a function $\mathbb{Q}^{t} \longrightarrow \mathbb{Q}$ s.t.

$$
\operatorname{FFYWG}_{\alpha}\left(\mathscr{F}_{1}, \mathscr{F}_{2}, \ldots, \mathscr{F}_{t}\right)=\bigotimes_{b=1}^{t} \mathscr{F}_{b}^{\alpha_{b}},
$$

where $\alpha=\left(\alpha_{1}, \alpha_{2}, \ldots, \alpha_{t}\right)^{T}$ is WV of $\mathscr{F}_{b}$ with $\alpha_{b}>0$ and $\sum_{b=1}^{t} \alpha_{b}=1$.

Theorem 12. The aggregated value by applying the FFYWG operator is also an FFN and given by 


$$
\operatorname{FFYWG}_{\alpha}\left(\mathscr{F}_{1}, \mathscr{F}_{2}, \ldots, \mathscr{F}_{t}\right)=\left\langle\sqrt[3]{1-\min \left(1,\left(\sum_{b=1}^{t}\left(\alpha_{b}\left(1-\mu_{b}^{3}\right)^{\eta}\right)\right)^{1 / \eta}\right)}, \sqrt[3]{\min \left(1,\left(\sum_{b=1}^{t}\left(\alpha_{b} \nu_{b}^{3 \eta}\right)\right)^{1 / \eta}\right)}\right\rangle .
$$

Proof. It is similar to Theorem 2.

Example 4. Consider Example 2, and by Theorem 12, the aggregated value for FFNs is

$$
\begin{aligned}
\operatorname{FFYWG}_{\alpha}\left(\mathscr{F}_{1}, \mathscr{F}_{2}, \mathscr{F}_{3}, \mathscr{F}_{4}\right)= & \left\langle\sqrt[3]{1-\min \left(1,\left(\sum_{b=1}^{4}\left(\alpha_{b}\left(1-\mu_{b}^{3}\right)^{\eta}\right)\right)^{1 / \eta}\right)}, \sqrt[3]{\min \left(1,\left(\sum_{b=1}^{4}\left(\alpha_{b} v_{b}^{3 \eta}\right)\right)^{1 / \eta}\right)}\right\rangle \\
= & \left\langle\sqrt[3]{1-\min \left(1,\left(0.2\left(1-0.6^{3}\right)^{2}+0.3\left(1-0.8^{3}\right)^{2}+0.2\left(1-0.7^{3}\right)^{2}+0.3\left(1-0.9^{3}\right)^{2}\right)^{1 / 2}\right)}\right. \\
& \left.\sqrt[3]{\min \left(1,\left(0.2(0.9)^{6}+0.3(0.7)^{6}+0.2(0.6)^{6}+0.3(0.7)^{6}\right)^{1 / 2}\right)}\right\rangle \\
= & \langle 0.77,0.76\rangle
\end{aligned}
$$

We give some statements without the proof.

Theorem 13 (idempotency). If all FFNs are identical, i.e., $\mathscr{F}_{b}=\mathscr{F}$, then

$$
\operatorname{FFYWG}\left(\mathscr{F}_{1}, \mathscr{F}_{2}, \ldots, \mathscr{F}_{t}\right)=\mathscr{F} .
$$

Theorem 14 (boundedness). Let $\mathscr{F}_{b}=\left(\mu_{b}, v_{b}\right)$ be a number of FFNs. Let $\mathscr{F}^{-}=\min \left(\mathscr{F}_{1}, \mathscr{F}_{2}, \ldots, \mathscr{F}_{t}\right)$ and $\mathscr{F}^{+}=\max \left(\mathscr{F}_{1}, \mathscr{F}_{2}, \ldots, \mathscr{F}_{t}\right)$. Then

$$
\mathscr{F}^{-} \leq \operatorname{FFYWG}\left(\mathscr{F}_{1}, \mathscr{F}_{2}, \ldots, \mathscr{F}_{t}\right) \leq \mathscr{F}^{+} .
$$

Theorem 15 (monotonicity). Consider two collections $\mathscr{F}_{b}^{\prime}=$ $\left\{\mathscr{F}_{1}^{\prime}, \mathscr{F}_{2}^{\prime}, \ldots, \mathscr{F}_{t}^{\prime}\right\}$ and $\mathscr{F}_{b}=\left\{\mathscr{F}_{1}, \mathscr{F}_{2}, \ldots, \mathscr{F}_{t}\right\}$ of FFNs. If $\mu_{b}^{\prime} \leq \mu_{b}$ and $\nu_{b}^{\prime} \geq v_{b}, \forall b$. Then,

$$
\operatorname{FFYWG}\left(\mathscr{F}_{1}^{\prime}, \mathscr{F}_{2}^{\prime}, \ldots, \mathscr{F}_{t}^{\prime}\right) \leq \operatorname{FFYWG}\left(\mathscr{F}_{1}, \mathscr{F}_{2}, \ldots, \mathscr{F}_{t}\right) \text {. }
$$

Theorem 16 (reducibility). Let $\mathscr{F}_{b}=\left(\mu_{b}, v_{b}\right)(b=$ $1,2, \ldots, t)$ be a collection of FFNs with WV $\alpha=\left(\alpha_{1}, \alpha_{2}, \ldots, \alpha_{t}\right)^{T}=(1 / t, 1 / t, \ldots, 1 / t)^{T}$. Then, FFYWG operator is

$$
\operatorname{FFYWG}_{\alpha}\left(\mathscr{F}_{1}, \mathscr{F}_{2}, \ldots, \mathscr{F}_{t}\right)=\left\langle\sqrt[3]{1-\min \left(1, \frac{1}{t}\left(\sum_{b=1}^{t}\left(\left(1-\mu_{b}^{3}\right)^{\eta}\right)\right)^{1 / \eta}\right)}, \sqrt[3]{\min \left(1, \frac{1}{t}\left(\sum_{b=1}^{t}\left(v_{b}^{3 \eta}\right)\right)^{1 / \eta}\right)}\right\rangle .
$$

We now define FFYOWG operators.

Definition 11. Let $\mathscr{F}_{b}=\left\langle\mu_{b}, v_{b}\right\rangle(b=1,2, \ldots, t)$ be a collection of FFNs with WV $\alpha=\left(\alpha_{1}, \alpha_{2}, \ldots, \alpha_{t}\right)^{T}$ with $\alpha_{b}>0$ and $\sum_{b=1}^{t} \alpha_{b}=1$. The FFYOWG operator is a function $\mathbb{Q}^{t} \longrightarrow \mathbb{Q}$ s.t.

$$
\operatorname{FFYOWG}_{\alpha}\left(\mathscr{F}_{1}, \mathscr{F}_{2}, \ldots, \mathscr{F}_{t}\right)=\bigotimes_{b=1}^{t}\left(\mathscr{F}_{\varrho(b)}\right)^{\alpha_{b}},
$$

where $(\varrho(1), \varrho(2), \ldots, \varrho(t)) \quad$ is permutation of $(b=1,2, \ldots, t)$ s.t. $\mathscr{F}_{\varrho(b-1)} \geq \mathscr{F}_{\varrho(b)}, \forall b=1,2, \ldots, t$.

Theorem 17. The aggregated value by applying the FFYOWG operator is also an FFN and given by

$$
\operatorname{FFYOWG}_{\alpha}\left(\mathscr{F}_{1}, \mathscr{F}_{2}, \ldots, \mathscr{F}_{t}\right)=\left\langle\sqrt[3]{1-\min \left(1,\left(\sum_{b=1}^{t}\left(\alpha_{b}\left(1-\mu_{\varrho(b)}^{3}\right)^{\eta}\right)\right)^{1 / \eta}\right)}, \sqrt[3]{\min \left(1,\left(\sum_{b=1}^{t}\left(\alpha_{b} v_{\varrho(b)}^{3 \eta}\right)\right)^{1 / \eta}\right)}\right\rangle .
$$


Proof. It is similar to Theorem 2.

Example 5. Consider Example 3, and by Theorem 17, the clumped value for FFNs is

$$
\begin{aligned}
\operatorname{FFYOWG}_{\alpha}\left(\mathscr{F}_{1}, \mathscr{F}_{2}, \mathscr{F}_{3}, \mathscr{F}_{4}\right) & =\bigotimes_{b=1}^{4}\left(\mathscr{F}_{\varrho(b)}\right)^{\alpha_{b}} \\
& \left.\left.\left.=\left\langle\sqrt[3]{1-\min \left(1,\left(\sum_{b=1}^{4}\left(\alpha_{b}\left(1-\mu_{\varrho(b)}^{3}\right)^{\eta}\right)\right)^{1 / \eta}\right.}\right), \sqrt[3]{\min \left(1,\left(\sum_{b=1}^{4}\left(\alpha_{b} \nu_{\varrho(b)}^{3 \eta}\right)\right)\right.}\right\rangle\right)^{1 / \eta}\right) \\
& =\left\langle\sqrt[3]{1-\min \left(1,\left(0.1\left(1-0.8^{3}\right)^{2}+0.3\left(1-0.6^{3}\right)^{2}+0.3\left(1-0.6^{3}\right)^{2}+0.3\left(1-0.9^{3}\right)^{2}\right)^{1 / 2}\right)}\right. \\
& =\langle 0.71,0.71\rangle .
\end{aligned}
$$

Remark 2. FFYOWG operators satisfy the properties (31), (33), (40), and (42).

Now, we define FFYHWG operators.
Definition 12. An FFYHWG operator is a function $\mathbb{Q}^{t} \longrightarrow Q$, with correlated WV $\alpha=\left(\alpha_{1}, \alpha_{2}, \ldots, \alpha_{t}\right)^{T}$ with $\alpha_{b}>0$ and $\sum_{b=1}^{t} \alpha_{b}=1$ s.t.

$$
\begin{aligned}
\operatorname{FFYHWG}_{\alpha}\left(\mathscr{F}_{1}, \mathscr{F}_{2}, \ldots, \mathscr{F}_{t}\right) & =\bigotimes_{b=1}^{t}\left(\dot{\mathscr{F}}_{\varrho(b)}\right)^{\alpha_{b}} \\
& =\left\langle\sqrt[3]{1-\min \left(1,\left(\sum_{b=1}^{t}\left(\alpha_{b}\left(1-\dot{\mu}_{\varrho(b)}^{3}\right)^{\eta}\right)\right)^{1 / \eta}\right)}, \sqrt[3]{\min \left(1,\left(\sum_{b=1}^{t}\left(\alpha_{b} \dot{v}_{\varrho(b)}^{3 \eta}\right)\right)^{1 / \eta}\right)}\right\rangle
\end{aligned}
$$

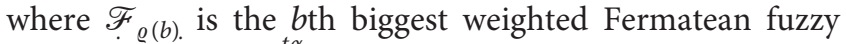
values $\dot{\mathscr{F}}_{b}\left(\dot{\mathscr{F}}_{b}=\mathscr{F}_{b}^{t \alpha_{b}}, b=1,2, \ldots, t\right)$.

\section{MADM Problems under Proposed Operators}

In this section, based on the FFYWA (or FFYWG) operator, we propose an MADM problem with FFNs. Let $\mathscr{L}=\left\{\mathscr{L}_{1}, \mathscr{L}_{2}, \ldots, \mathscr{L}_{m}\right\}$ be a set of alternatives and $\alpha=\left\{\alpha_{1}, \alpha_{2}, \ldots, \alpha_{t}\right\} \quad$ is WV of the attributes $\mathscr{K}=\left\{\mathscr{K}_{1}, \mathscr{K}_{2}, \ldots, \mathscr{K}_{t}\right\}$, where $\alpha_{b}>0$ and $\sum_{b=1}^{t} \alpha_{b}=1$. Suppose that $\widetilde{\mathscr{E}}=\left(\mu_{l b}, v_{l b}\right)_{m \times t}$ is the FF decision matrix, where $\mu_{l b}$ and $\nu_{l b}$ are satisfaction and dissatisfaction degrees of the alternative for the attribute given by the decision maker (DMr), where $0 \leq \mu_{l b}^{3}+\nu_{l b}^{3} \leq 1$.

For solving an MADM problem, Algorithm 1 is given as follows.

4.1. Selection of an Authentic Lab for the COVID-19 Test. COVID-19 is a pandemic disease. "CO" stands for Corona, "VI" stands for Virus, and "D" stands for Disease. Simply, this disease is called "CoronaVirus 2019." Most of the infected people with the COVID-19 virus suffer respiratory illness, and some recover without requiring special treatment. Older people and people having diseases like cardiovascular disease, diabetes, chronic respiratory disease, and cancer are more likely to develop serious illness. Mostly COVID-19 spreads through the sneezing or coughing of an infected person. So, it is necessary to follow respiratory etiquette, for example, by coughing into a flexed elbow. Nowadays, definite vaccines or treatments are available to treat COVID-19 patients. However, the scientists and pharmacists are working hard to evaluate potential treatments through clinical trials.

Different countries are trying to control and reduce the spread of this virus by quarantining citizens, limiting travel, testing and treating patients, carrying out contact tracing, and canceling large gatherings. This pandemic is moving like a waveone that infected rapidly many people in a few months. We can say that COVID-19 is much more than a health crisis. COVID-19 is affecting adversely the economic, social, and politically condition of every country all over the world.

In such a critical situation of COVID-19, it is mandatory that people who have the symptoms of this virus must go for the medical test. For this purpose, we required an authentic lab. The main motive of this application is to select the authentic lab from different labs for the COVID-19 test by applying FFYWA and FFYWG operators. Let $\mathscr{L}=\left\{\mathscr{L}_{1}, \mathscr{L}_{2}, \mathscr{L}_{3}, \mathscr{L}_{4}, \mathscr{L}_{5}\right\}$ be a set of labs. Let $\mathscr{K}=\left\{\mathscr{K}_{1}, \mathscr{K}_{2}, \mathscr{K}_{3}\right\}$ be a set of three attributes for the 
(1) Input:

$\mathscr{L}:$ Set of alternatives,

$\mathscr{K}$ : Set of attributes,

$\alpha$ : WV for alternatives.

(2) Use the FFYWA (or FFYWG) operator to evaluate the information in the FF decision matrix, and find preference values $\mathscr{L}_{l}, l=1,2, \ldots, m$, of the alternatives $\mathscr{L}_{l}$.

$$
\begin{aligned}
& \mathscr{L}_{l}=\operatorname{FFYWA}\left(\mathscr{L}_{l 1}, \mathscr{L}_{l 2}, \ldots, \mathscr{L}_{l t}\right) \\
& =\left\langle\sqrt[3]{\min \left(1,\left(\sum_{b=1}^{t}\left(\alpha_{b} \mu_{b}^{3 \eta}\right)\right)^{1 / \eta}\right)}, \sqrt[3]{1-\min \left(1,\left(\sum_{b=1}^{t}\left(\alpha_{b}\left(1-v_{b}^{3}\right)^{\eta}\right)\right)^{1 / \eta}\right)}\right\rangle . \\
& \text { OR } \\
& \mathscr{q}_{l}=\operatorname{FFYWG}\left(\mathscr{L}_{l 1}, \mathscr{L}_{l 2}, \ldots, \mathscr{L}_{l t}\right) \\
& =\left\langle\sqrt[3]{1-\min \left(1,\left(\sum_{b=1}^{t}\left(\alpha_{b}\left(1-\mu_{b}^{3}\right)^{\eta}\right)\right)^{1 / \eta}\right)}, \sqrt[3]{\min \left(1,\left(\sum_{b=1}^{t}\left(\alpha_{b} v_{b}^{3 \eta}\right)\right)^{1 / \eta}\right)}\right\rangle .
\end{aligned}
$$

(3) Compute the score values.

(4) Use the score values $S\left(\mathscr{g}_{l}\right)$ to rank the alternatives $\mathscr{L}_{l}, l=1,2, \ldots, m$. For equal score, use the accuracy function for ranking of alternatives.

Output: The alternative with greatest score will be the decision.

Algorithm 1: Steps to deal with an MADM problem by the FFYWA (or FFYWG) operator.

evaluation of the lab, with weights as $\alpha_{1}=0.3, \alpha_{2}=0.4$, and $\alpha_{3}=0.3$, where $\mathscr{K}_{1}$ represents time limits, $\mathscr{K}_{2}$ represents accurate results, and $\mathscr{K}_{3}$ represents location flexibility for the client. The decision matrix of such an alternative is given in Table 1.

Then, we proceed to select the suitable alternative by the FFYWA operator by implementing the steps mentioned in Algorithm 1.

Step 1 . The performance values $q_{\ell}$ of the alternatives by the FFYWA operator for $\eta=3$ are

$$
\begin{aligned}
& \mathscr{q}_{1}=(0.72,0.34), \\
& \mathscr{q}_{2}=(0.72,0.48), \\
& \mathscr{q}_{3}=(0.58,0.56), \\
& \mathscr{q}_{4}=(0.87,0.40), \\
& \mathscr{q}_{5}=(0.53,0.27) .
\end{aligned}
$$

Step 2. The scores $S\left(g_{\ell}\right)$ of all FFNs are

$$
\begin{aligned}
& S\left(g_{1}\right)=0.33, \\
& S\left(g_{2}\right)=0.26, \\
& S\left(g_{3}\right)=0.02, \\
& S\left(g_{4}\right)=0.59, \\
& S\left(g_{5}\right)=0.13 .
\end{aligned}
$$

Step 3. Ranking of alternatives according to scores $S\left(g_{\ell}\right)$ is

$$
\mathscr{L}_{4}>\mathscr{L}_{1}>\mathscr{L}_{2}>\mathscr{L}_{5}>\mathscr{L}_{3}
$$

TABLE 1: FF decision matrix.

\begin{tabular}{lccccc}
\hline$\widetilde{\mathscr{E}}$ & $\mathscr{L}_{1}$ & $\mathscr{L}_{2}$ & $\mathscr{L}_{3}$ & $\mathscr{L}_{4}$ & $\mathscr{L}_{5}$ \\
\hline $\mathscr{K}_{1}$ & $(0.7,0.4)$ & $(0.8,0.6)$ & $(0.5,0.3)$ & $(0.7,0.5)$ & $(0.6,0.1)$ \\
$\mathscr{K}_{2}$ & $(0.6,0.3)$ & $(0.7,0.5)$ & $(0.6,0.8)$ & $(0.9,0.3)$ & $(0.4,0.1)$ \\
$\mathscr{K}_{3}$ & $(0.8,0.3)$ & $(0.5,0.2)$ & $(0.6,0.4)$ & $(0.9,0.4)$ & $(0.3,0.4)$ \\
\hline
\end{tabular}

Step 4. Thus, we conclude that $\mathscr{L}_{4}$ is the most authentic lab for the COVID-19 test.

On the other hand, if the FFYWG operator is used to solve the same MADM problem, the following steps lead us to choose the optimal alternative:

Step 1. The performance values $\mathscr{Q}_{\ell}$ of the alternatives by the FFYWG operator for $\varphi=3$ are

$$
\begin{aligned}
& q_{1}=(0.68,0.36), \\
& q_{2}=(0.67,0.54), \\
& q_{3}=(0.57,0.72), \\
& q_{4}=(0.81,0.44), \\
& q_{5}=(0.45,0.35) .
\end{aligned}
$$

Step 2. The scores $S\left(g_{\ell}\right)$ of all FFNs are

$$
\begin{aligned}
& S\left(g_{1}\right)=0.27 \\
& S\left(g_{2}\right)=0.14 \\
& S\left(g_{3}\right)=-0.19 \\
& S\left(g_{4}\right)=0.45 \\
& S\left(g_{5}\right)=0.05
\end{aligned}
$$

Step 3. Ranking of alternatives according to scores $S\left(g_{\ell}\right)$ is

$$
\mathscr{L}_{4}>\mathscr{L}_{1}>\mathscr{L}_{2}>\mathscr{L}_{5}>\mathscr{L}_{3}
$$


TABLE 2: RO using the FFYWA operator.

\begin{tabular}{ccccccc}
\hline$\eta$ & $S\left(\mathscr{g}_{1}\right)$ & $S\left(g_{2}\right)$ & $S\left(g_{3}\right)$ & $S\left(g_{4}\right)$ & $S\left(g_{5}\right)$ & $\mathrm{RO}$ \\
\hline 1 & 0.30 & 0.21 & -0.04 & 0.55 & 0.08 & $\mathscr{L}_{4}>\mathscr{L}_{1}>\mathscr{L}_{2}>\mathscr{L}_{5}>\mathscr{L}_{3}$ \\
2 & 0.32 & 0.25 & 0.00 & 0.57 & 0.10 & $\mathscr{L}_{4}>\mathscr{L}_{1}>\mathscr{L}_{2}>\mathscr{L}_{5}>\mathscr{L}_{3}$ \\
3 & 0.33 & 0.26 & 0.02 & 0.59 & 0.13 & $\mathscr{L}_{4}>\mathscr{L}_{1}>\mathscr{L}_{2}>\mathscr{L}_{5}>\mathscr{L}_{3}$ \\
4 & 0.37 & 0.30 & 0.05 & 0.62 & 0.14 & $\mathscr{L}_{4}>\mathscr{L}_{1}>\mathscr{L}_{2}>\mathscr{L}_{5}>\mathscr{L}_{3}$ \\
5 & 0.39 & 0.32 & 0.07 & 0.62 & 0.15 & $\mathscr{L}_{4}>\mathscr{L}_{1}>\mathscr{L}_{2}>\mathscr{L}_{5}>\mathscr{L}_{3}$ \\
\hline
\end{tabular}

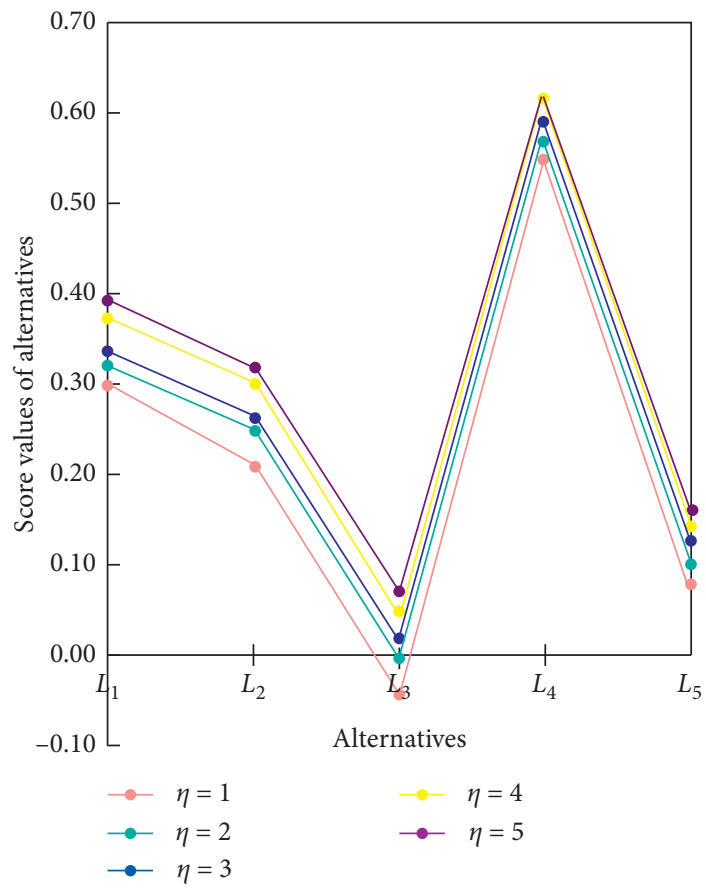

FIGURE 1: RO using the FFYWA operator.

Step $4 . \mathscr{L}_{4}$ is the best alternative.

4.2. Effect of Different Parameter's Values. Parameter $\varphi$ plays an essential role in the ranking results. Based on FFYWA and FFYWG operators, the effect of parameter $\varphi$ on the score functions and ranking results is investigated in this section. To explain an influence of parameter $\varphi$ on the score functions and ranking results, we discuss the effects from the following two ways:

(1) We examine the effect of parameter $\varphi$ on the ranking results of alternatives by using the FFYWA operator.

(2) We examine the effect of parameter $\varphi$ on the ranking results of alternatives by using the FFYWG operator.

It is clear from Table 2 and Figure 1 that by using the FFYWA operator, score values of the alternatives are different or same but increase as we increase the value of $\varphi$ and the ranking order (RO) is same. Similarly, in the FFYWG operator, score values of the alternatives are different or same but decrease as we increase the value of $\varphi$ and the RO is same as shown in Table 3 and Figure 2. Hence, it is clear that in the FFYWA operator, score values increase but in the FFYWG operator, score values decrease. However, the ranking result is same.

\section{Comparison Analysis and Discussion}

5.1. Comparison with Pythagorean Fuzzy Yager Aggregation Operators. To compute performance and validity of our proposed operators, here we aggregate the same data using different operators, namely, Pythagorean fuzzy Yager weighted average (PFYWA) [14] and Pythagorean fuzzy Yager weighted geometric (PFYWG) [14] operators. The computed results by applying these operators are summarized in Table 4 and shown in Figure 3.

It is clear from Table 4 and Figure 3 that the best alternative obtained by using PFYWA and PFYWG operators remains same as obtained from using the proposed operators. This implies that our proposed methods are authentic and can be applied in DM problems. The main logic behind our proposed approach is that the PFS handles the situation where $\mu^{2}+v^{2} \leq 1$ but fails in situations where $\mu^{3}+v^{3} \leq 1$. If we assign MD 0.8 and NMD 0.7, then the proposed operators in [14] fail to cope the situation. That is why, we need the FFS and our proposed theory.

5.2. Fermatean Fuzzy Yager Aggregation Operators and Fermatean Fuzzy TOPSIS Method. In this section, we discuss the steps to solve any MADM problem by FF Yager AOs and the FF TOPSIS method in Figure 4. 
TABLE 3: RO using the FFYWG operator.

\begin{tabular}{ccccccc}
\hline$\eta$ & $S\left(\mathscr{g}_{1}\right)$ & $S\left(\mathscr{q}_{2}\right)$ & $S\left(\mathscr{g}_{3}\right)$ & $S\left(\mathscr{q}_{4}\right)$ & $S\left(g_{5}\right)$ & $\mathrm{RO}$ \\
\hline 1 & 0.30 & 0.20 & -0.04 & 0.55 & 0.08 & $\mathscr{L}_{4}>\mathscr{L}_{1}>\mathscr{L}_{2}>\mathscr{L}_{5}>\mathscr{L}_{3}$ \\
2 & 0.29 & 0.17 & -0.14 & 0.49 & 0.06 & $\mathscr{L}_{4}>\mathscr{L}_{1}>\mathscr{L}_{2}>\mathscr{L}_{5}>\mathscr{L}_{3}$ \\
3 & 0.27 & 0.14 & -0.19 & 0.45 & 0.05 & $\mathscr{L}_{4}>\mathscr{L}_{1}>\mathscr{L}_{2}>\mathscr{L}_{5}>\mathscr{L}_{3}$ \\
4 & 0.27 & 0.11 & -0.22 & 0.42 & 0.04 & $\mathscr{L}_{4}>\mathscr{L}_{1}>\mathscr{L}_{2}>\mathscr{L}_{5}>\mathscr{L}_{3}$ \\
5 & 0.25 & 0.09 & -0.24 & 0.38 & 0.03 & $\mathscr{L}_{4}>\mathscr{L}_{1}>\mathscr{L}_{2}>\mathscr{L}_{5}>\mathscr{L}_{3}$ \\
\hline
\end{tabular}

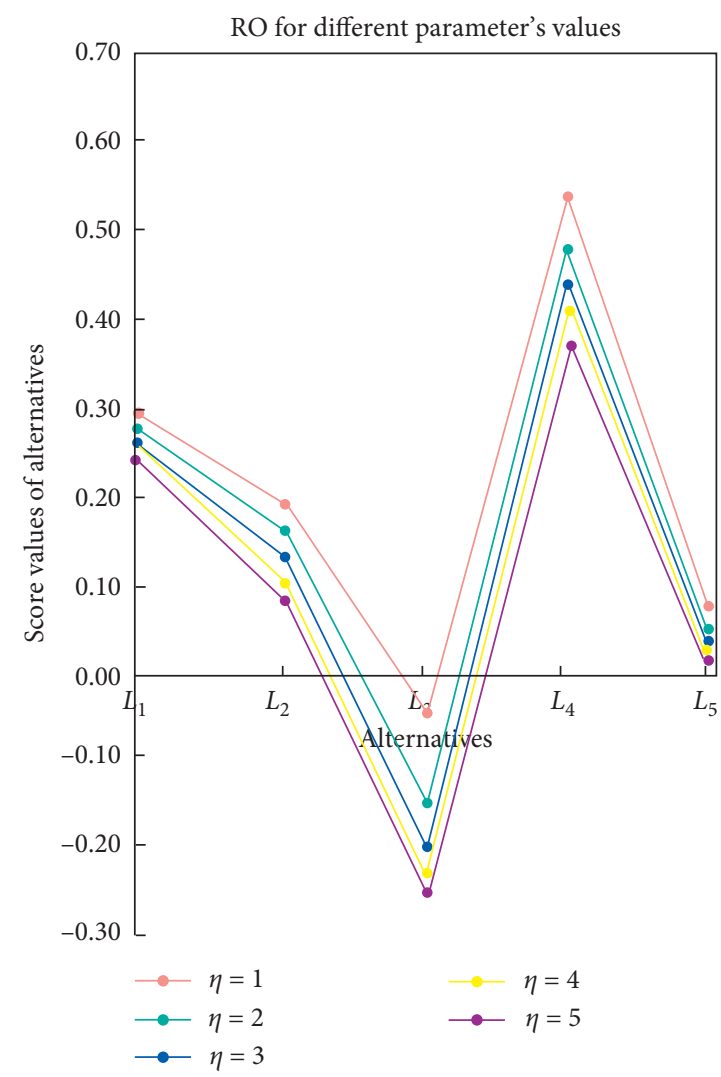

FIgURE 2: RO using the FFYWG operator.

TABLE 4: Comparison analysis with the existing operators.

\begin{tabular}{lcccccc}
\hline Methods & $S\left(\mathscr{q}_{1}\right)$ & $S\left(\mathscr{q}_{2}\right)$ & $S\left(\mathscr{q}_{3}\right)$ & $S\left(\mathscr{q}_{4}\right)$ & $S\left(\mathscr{q}_{5}\right)$ & $\mathrm{RO}$ \\
\hline FFYWA & 0.33 & 0.26 & 0.02 & 0.59 & 0.13 & $\mathscr{L}_{4}>\mathscr{L}_{1}>\mathscr{L}_{2}>\mathscr{L}_{5}>\mathscr{L}_{3}$ \\
FFYWG & 0.27 & 0.14 & -0.19 & 0.45 & 0.05 & $\mathscr{L}_{4}>\mathscr{L}_{1}>\mathscr{L}_{2}>\mathscr{L}_{5}>\mathscr{L}_{3}$ \\
PFYWA & 0.41 & 0.29 & 0.09 & 0.52 & 0.26 & $\mathscr{L}_{4}>\mathscr{L}_{1}>\mathscr{L}_{2}>\mathscr{L}_{5}>\mathscr{L}_{3}$ \\
PFYWG & 0.35 & 0.15 & -0.16 & 0.41 & 0.14 & $\mathscr{L}_{4}>\mathscr{L}_{1}>\mathscr{L}_{2}>\mathscr{L}_{5}>\mathscr{L}_{3}$ \\
\hline
\end{tabular}

Here, we discuss the comparison of our proposed theory with the Fermatean fuzzy TOPSIS method [5]. The steps to find out the best alternative in Application 4.1 by the Fermatean fuzzy TOPSIS method are as follows:

(1) Table 1 represents the FF decision matrix in which each entry corresponds to an FFN.

(2) The FF positive ideal solution FFPIS $\mathcal{S}^{+}$and FF negative ideal solution FFNIS $\mathcal{S}^{-}$are given as

$$
\begin{aligned}
& \mathcal{S}^{+}=\{(0.8,0.6),(0.9,0.3),(0.9,0.4)\} \\
& \mathcal{S}^{-}=\{(0.5,0.3),(0.6,0.8),(0.3,0.4)\}
\end{aligned}
$$

(3) The distance between the alternative $\mathscr{L}_{\ell}$ and FFPIS $\mathcal{S}^{+}$together with the FFNIS $\mathcal{S}^{-}$is given in Table 5 .

(4) The revised closeness degree of each alternative is given as 


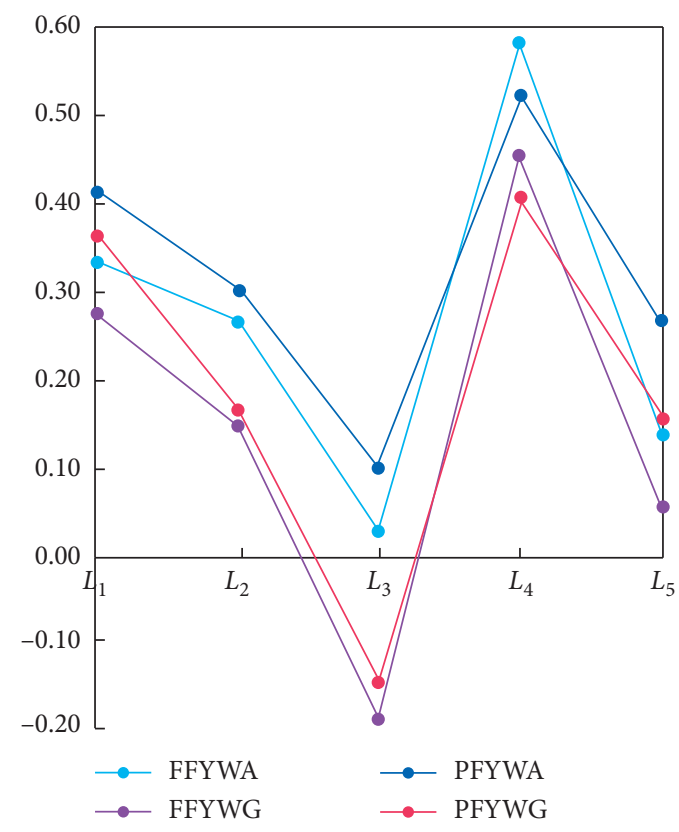

FIgURE 3: Comparison of the four methods.

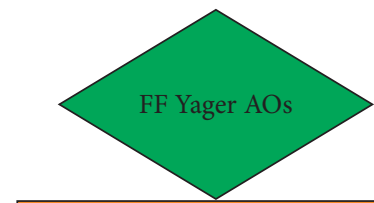

FF decision matrix

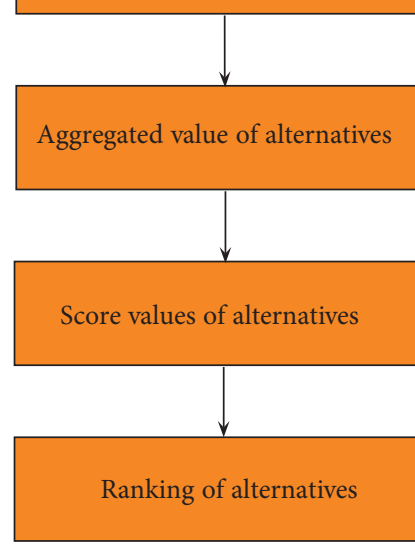

Ranking of alternatives

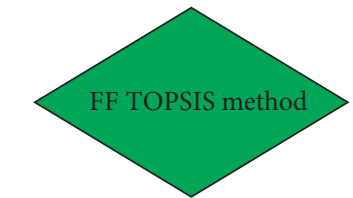

FF decision matrix

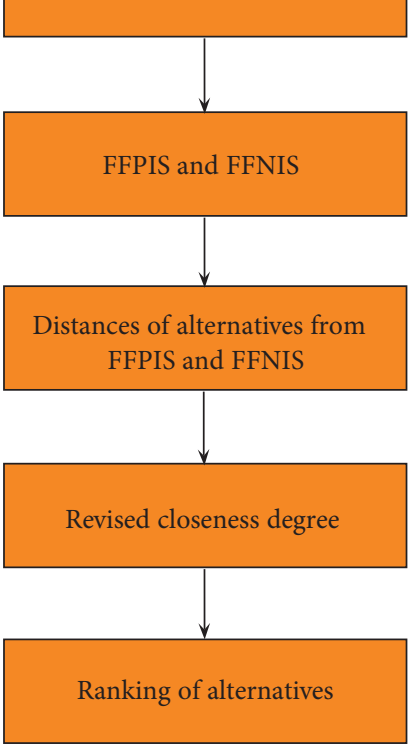

(b)

FIgURE 4: Flow chart for the MADM problem using (a) FF Yager AOs and (b) the FF TOPSIS method. 
TABLE 5: Distance of alternatives from FFPIS and FFNIS.

\begin{tabular}{lc}
\hline$D\left(\mathscr{L}_{\ell}, \mathcal{S}^{+}\right)$ & $D\left(\mathscr{L}_{\ell}, \mathcal{S}^{-}\right)$ \\
\hline 0.37 & 0.4 \\
0.33 & 0.32 \\
0.5 & 0.06 \\
0.07 & 0.5 \\
0.61 & 0.27 \\
\hline
\end{tabular}

$$
\begin{aligned}
& \xi\left(\mathscr{L}_{1}\right)=-4.49 \\
& \xi\left(\mathscr{L}_{2}\right)=-4.07 \\
& \xi\left(\mathscr{L}_{3}\right)=-7.02 \\
& \xi\left(\mathscr{L}_{4}\right)=0 \\
& \xi\left(\mathscr{L}_{5}\right)=-8.17
\end{aligned}
$$

(5) We get the following ranking list by arranging the alternatives in ascending order with respect to $\xi\left(\mathscr{L}_{l}\right)$ :

$$
\mathscr{L}_{4}>\mathscr{L}_{2}>\mathscr{L}_{1}>\mathscr{L}_{3}>\mathscr{L}_{5}
$$

(6) $\mathscr{L}_{4}$ is the best alternative.

From the outcomes of the proposed operators and the FF TOPSIS method, we conclude that ranking lists obtained from both compared methods are slightly different, but the best alternative from both approaches is same. The FF TOPSIS method is a good approach to solve DM problems, but there are many hindrances which can be solved by using our proposed theory. The FF Yager AOs are a more flexible and easy approach. The best alternative can be obtained by a short process. The results from the proposed theory are more accurate and closest to original results. However, in FF Yager AOs, we can also discuss the effect of parameters.

\section{Conclusions}

FFS has more general structure that represents an extension of IFS and PFS and satisfies the condition $\mu^{3}+\nu^{3} \leq 1$ and is more flexible to solve decision-making problems involving vagueness. Moreover, Yager's t-norm and t-conorm have more generalized structure that operates efficiently to integrate the intricate information. The shortcomings of the existing methods and beneficial characteristics of Yager AOs motivate us to endeavor for the development of a fruitful fusion with FFNs.

In this article, we have modified the multiskilled Yager AOs under the FF model to combine the advantages and flexibility of both theories. Later, we have thrown light on elementary operations of FFNs to construct Fermatean fuzzy AOs that obey the principles of Yager operations. We have established the FFYWA, FFYOWA, FFYHWA, FFYWG, FFYOWG, and FFYHWG operators to aggregate the FFNs. We have explored some main properties of the proposed operators including idempotency, boundedness, monotonicity, and reducibility.
The foremost aim of this study is to present a strategy based on the proposed operators to tackle MADM comprising FF assessments. As a preparation to their utilization in MADM, theoretical basis of AOs needs to be carefully considered. A practical example for the selection of a suitable lab for the COVID-19 test is given to demonstrate the application of the proposed strategy. We have performed the comparison analysis of our proposed theory with the existing operators as well as the FF TOPSIS method. We have highlighted the superiorities of our proposed operators over the FF TOPSIS method and other existing operators. We have examined the effect of various values of parameters on the outcomes of MADM problems. In short, this article builds up a tool that has the rich properties of Yager AOs and flexibility of the FF model. In future research, we will extend our models to Fermatean fuzzy soft set environment.

\section{Data Availability}

No data were used to support this study.

\section{Conflicts of Interest}

The authors declare no conflicts of interest.

\section{References}

[1] L. A. Zadeh, "Fuzzy sets," Information and Control, vol. 8, no. 3, pp. 338-353, 1965.

[2] K. T. Atanassov, "Intuitionistic fuzzy sets," Fuzzy Sets And Systems, vol. 20, no. 1, pp. 87-96, 1986.

[3] R. R. Yager, "Pythagorean fuzzy subsets," in Proceedings of the 2013 Joint IFSA World Congress and NAFIPS Annual Meeting (IFSA/NAFIPS), pp. 57-61, IEEE, Edmonton, Canada, June 2013.

[4] R. R. Yager, "Generalized orthopair fuzzy sets," IEEE Transactions on Fuzzy Systems, vol. 25, no. 5, pp. 1222-1230, 2016.

[5] T. Senapati and R. R. Yager, "Fermatean fuzzy sets," Journal of Ambient Intelligence And Humanized Computing, vol. 11, no. 2, pp. 663-674, 2020.

[6] Z. Xu, "Intuitionistic fuzzy aggregation operators," IEEE Transactions on Fuzzy Systems, vol. 15, no. 6, pp. 1179-1187, 2007.

[7] Z. Xu and R. R. Yager, "Some geometric aggregation operators based on intuitionistic fuzzy sets," International Journal of General Systems, vol. 35, no. 4, pp. 417-433, 2006.

[8] D.-F. Li, "Multiattribute decision making method based on generalized OWA operators with intuitionistic fuzzy sets," Expert Systems With Applications, vol. 37, no. 12, pp. 86738678, 2010.

[9] G. Wei, "Some induced geometric aggregation operators with intuitionistic fuzzy information and their application to group decision making," Applied Soft Computing, vol. 10, no. 2, pp. 423-431, 2010.

[10] R. R. Yager, "Pythagorean membership grades in multi-criteria decision making," IEEE Transactions on Fuzzy Systems, vol. 22, no. 4, pp. 958-965, 2013.

[11] X. Peng and Y. Yang, "Fundamental properties of intervalvalued Pythagorean fuzzy aggregation operators," International Journal of Intelligent Systems, vol. 31, no. 5, pp. 444-487, 2016. 
[12] S. Zeng, J. Chen, and X. Li, "A hybrid method for Pythagorean fuzzy multiple-criteria decision making," International Journal of Information Technology \& Decision Making, vol. 15, no. 2, pp. 403-422, 2016.

[13] M. Akram, W. A. Dudek, and J. M. Dar, "Pythagorean Dombi fuzzy aggregation operators with application in multicriteria decision-making," International Journal of Intelligent Systems, vol. 34, no. 11, pp. 3000-3019, 2019.

[14] G. Shahzadi, M. Akram, and A. N. Al-Kenani, "Decisionmaking approach under Pythagorean fuzzy yager weighted operators," Mathematics, vol. 8, no. 1, p. 70, 2020.

[15] M. Akram, W. A. Dudek, and F. Ilyas, "Group decisionmaking based on pythagorean fuzzy TOPSIS method," International Journal of Intelligent Systems, vol. 34, no. 7, pp. 1455-1475, 2019.

[16] M. Akram, F. Ilyas, and H. Garg, "Multi-criteria group decision making based on ELECTRE I method in pythagorean fuzzy information," Soft Computing, vol. 24, no. 5, pp. 3425-3453, 2020.

[17] M. Akram and A. Bashir, "Complex fuzzy ordered weighted quadratic averaging operators," Granular Computing, 2020.

[18] M. Akram, H. Garg, and K. Zahid, "Extensions of ELECTRE-I and TOPSIS methods for group decision-making under complex pythagorean fuzzy environment," Iranian Journal of Fuzzy Systems, vol. 17, no. 5, pp. 147-164, 2020.

[19] M. Akram, Shumaiza, and M. Arshad, "Bipolar fuzzy TOPSIS and bipolar fuzzy ELECTRE-I methods to diagnosis," Computational and Applied Mathematics, vol. 39, no. 1, p. 7, 2020.

[20] G. Deschrijver, C. Cornelis, and E. E. Kerre, "On the representation of intuitionistic fuzzy $t$-norms and $t$-conorms," IEEE Transactions on Fuzzy Systems, vol. 12, no. 1, pp. 45-61, 2004.

[21] G. Deschrijver and E. E. Kerre, "A generalization of operators on intuitionistic fuzzy sets using triangular norms and conorms," Notes on Intuitionistic Fuzzy Sets, vol. 8, no. 1, pp. 19-27, 2002.

[22] H. Garg, "A new generalized pythagorean fuzzy information aggregation using Einstein operations and its application to decision making," International Journal of Intelligent Systems, vol. 31, no. 9, pp. 886-920, 2016.

[23] H. Garg, "Generalized intuitionistic fuzzy interactive geometric interaction operators using Einstein $t$-norm and $t$ conorm and their application to decision making," Computers \& Industrial Engineering, vol. 101, pp. 53-69, 2016.

[24] H. Garg, "New exponential operational laws and their aggregation operators for interval-valued pythagorean fuzzy multicriteria decision-making," International Journal of Intelligent Systems, vol. 33, no. 3, pp. 653-683, 2018.

[25] D. Rani and H. Garg, "Complex intuitionistic fuzzy power aggregation operators and their applications in multi-criteria decision making," Expert Systems, vol. 35, no. 6, Article ID e12325, 2018.

[26] P. Liu and P. Wang, "Some $q$-rung orthopair fuzzy aggregation operators and their applications to multiple-attribute decision making," International Journal of Intelligent Systems, vol. 33, no. 2, pp. 259-280, 2018.

[27] C. Jana, G. Muhiuddin, and M. Pal, "Some Dombi aggregation of Q-rung orthopair fuzzy numbers in multiple-attribute decision making," International Journal of Intelligent Systems, vol. 34, no. 12, pp. 3220-3240, 2019.

[28] P. Liu, S. M. Chen, and P. Wang, "Multiple-attribute group decision making based on q-rung orthopair fuzzy power maclaurin symmetric mean operators," IEEE Transactions on Systems, Man, and Cybernetics: Systems, 2018.
[29] P. Liu and J. Liu, "Some q-rung orthopair fuzzy Bonferroni mean operators and their application to multi-attribute group decision making," International Journal of Intelligent Systems, vol. 33, no. 2, pp. 315-347, 2018.

[30] T. Senapati and R. R. Yager, "Some new operations over Fermatean fuzzy numbers and application of Fermatean fuzzy WPM in multiple criteria decision making," Informatica, vol. 30, no. 2, pp. 391-412, 2019.

[31] T. Senapati and R. R. Yager, "Fermatean fuzzy weighted averaging/geometric operators and its application in multicriteria decision-making methods," Engineering Applications of Artificial Intelligence, vol. 85, pp. 112-121, 2019.

[32] M. Akram, A. Habib, and J. C. R. Alcantud, "An optimization study based on Dijkstra algorithm for a network with trapezoidal picture fuzzy numbers, neural computing and applications," Neural Computing and Applications, 2020.

[33] M. Akram and A. Luqman, "Granulation of ecological networks under fuzzy soft environment," Soft Computing, vol. 24, no. 16, pp. 11867-11892, 2020.

[34] S. A. Edalatpanah, "Neutrosophic structured element," Expert Systems, Article ID e12542, 2020.

[35] S. A. Edalatpanah, "Data envelopment analysis based on triangular neutrosophic numbers," CAAI Transactions on Intelligence Technology, vol. 5, no. 2, pp. 94-98, 2020.

[36] H. Garg, "A novel trigonometric operation based q-rung orthopair fuzzy aggregation operator and its fundamental properties, neural computing and applications," Neural Computing and Applications, 2020.

[37] H. Garg and S.-M. Chen, "Multiattribute group decision making based on neutrality aggregation operators of q-rung orthopair fuzzy sets," Information Sciences, vol. 517, pp. 427-447, 2020.

[38] R. Kumar, S. A. Edalatpanah, S. Jha, and R. Singh, "A novel approach to solve Gaussian valued neutrosophic shortest path problems," Infinite Study, vol. 8, pp. 347-353, 2019.

[39] Z. Yang, X. Li, H. Garg, and M. Qi, "Decision support algorithm for selecting an antivirus mask over COVID-19 pandemic under spherical normal fuzzy environment," International Journal of Environmental Research and Public Health, vol. 17, no. 10, p. 3407, 2020. 